\section{Regards sur l'économie allemande}

Bulletin économique du CIRAC

$89 \mid 2008$

Varia

\title{
La mise en place du gouvernement à distance de l'assurance maladie
}

\section{Patrick Hassenteufel}

\section{Q OpenEdition}

\section{Journals}

Édition électronique

URL : http://journals.openedition.org/rea/2673

DOI : 10.4000/rea.2673

ISBN : 978-2-8218-0874-4

ISSN : 1965-0787

\section{Éditeur}

CIRAC

\section{Édition imprimée}

Date de publication : 1 décembre 2008

Pagination : 27-33

ISSN : 1156-8992

\section{Référence électronique}

Patrick Hassenteufel, « La mise en place du gouvernement à distance de l'assurance maladie », Regards sur l'économie allemande [En ligne], 89 | décembre 2008, mis en ligne le 01 décembre 2010, consulté le 01 mai 2019. URL : http://journals.openedition.org/rea/2673; DOI : 10.4000/rea.2673 


\section{La mise en place du gouvernement à distance de 1'assurance maladie}

\section{Patrick Hassenteufel}

En mars 2007, après près d'un an de discussion au sein de la grande coalition et de conflits avec les caisses et les médecins, le Bundestag a définitivement adopté la Loi de renforcement de la concurrence dans l'assurance maladie légale (GKV-Wettbewerbsstärkungsgesetz, GKV-WSG). Le cœur de cette nouvelle réforme du système d'assurance maladie allemand est la création d'un Fonds pour la santé (Gesundheitsfonds) dont l'entrée en fonctionnement est prévue pour le $1^{\text {er }}$ janvier 2009. Sa mise en œuvre suppose la détermination préalable d'un budget global pour l'ensemble du système d'assurance maladie en tenant compte du changement des règles de rémunération des médecins et des besoins financiers des hôpitaux, ce qui s'est concrétisé par plusieurs décisions importantes depuis cet été. La traduction dans les faits de cette loi pose deux questions sur l'évolution d'ensemble du système d'assurance maladie allemand: comment évolue la concurrence entre les caisses dans ce nouveau cadre et en quoi celui-ci correspond-il à un renforcement de la régulation par des autorités gouvernementales ?

Le vote, en mars 2007, de la Loi GKV-WSG correspond à un changement majeur dans la gouvernance et l'organisation du système du fait de la création d'un Un Fonds qui centralise l'ensemble des sources de financement... Fonds pour la santé : Gesundheitsfonds (voir REA 79/06). Celui-ci doit centraliser l'ensemble des sources de financement de l'assurance maladie : les cotisations des salariés et des employeurs ainsi que les ressources fiscales qui lui sont affectées. Par conséquent, ce ne sont plus les caisses paritaires (patronat, syndicats) qui gèrent directement les cotisations de leurs adhérents. Surtout, il en résulte la détermination par décret d'un taux unique de cotisation pour l'ensemble des caisses alors que, jusqu'alors, chaque caisse fixait son propre taux de cotisation.

Le fonctionnement du Gesundheitsfonds en 2009

\begin{tabular}{|c|c|c|}
\hline $\begin{array}{l}\text { Salarié } \\
\text { Cotisation sur le salaire brut }(7,3 \%) \\
\text { cotisation spéciale }(0,9 \% \text { du salaire brut) }\end{array}$ & $\begin{array}{l}\text { Employeur } \\
\text { Charges salariales sur le } \\
\text { salaire brut }(7,3 \%)\end{array}$ & $\begin{array}{l}\text { Etat } \\
\text { Impôts (4 milliards } €) \text {, } \\
\text { cotisations des retraités et des chômeurs }\end{array}$ \\
\hline 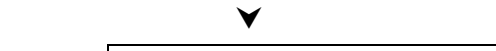 & $\checkmark$ & $V$ \\
\hline \multirow{2}{*}{\multicolumn{3}{|c|}{$\begin{array}{c}\text { Gesundheitsfonds } \\
\begin{array}{l}\text { Institution fédérale centralisant et répartissant selon un système de péréquation (RSA) } \\
\text { le budget global de l'assurance maladie (167 milliards } €)\end{array}\end{array}$}} \\
\hline & & \\
\hline \multicolumn{3}{|c|}{ Caisses (216) du système légal d'assurance maladie (GKV) } \\
\hline \multicolumn{3}{|c|}{$\begin{array}{c}\text { Elles perçoivent du Gesundheitsfonds un budget dont le montant dépend de l'âge, du sexe, de la profession des assurés (montan } \\
\text { forfaitaire mensuel par assuré, entre } 100 \text { et } 280 € \text { ), ainsi que de leur morbidité (montant complémentaire pour les patients aux } \\
\text { pathologies lourdes et chroniques) }\end{array}$} \\
\hline \multicolumn{3}{|c|}{$\begin{array}{l}\text { Remboursement aux assurés par les caisses excédentaires } \\
\text { Prime santé supplémentaire (8€ par mois maximum) demandée par les caisses déficitaires }\end{array}$} \\
\hline
\end{tabular}

Source : Ärzte Zeitung, 08-10-08. 
... et répartit les ressources entre les caisses selon une nouvelle péréquation

Trois enjeux principalement

Instauration d'un budget a priori

Un taux de cotisation unique de $15,5 \%$ du salaire brut
Ce Fonds doit aussi répartir les ressources financières entre les caisses dans le cadre d'un nouveau système de péréquation (Risikostrukturausgleich, $R S A$ ) basé sur le niveau de revenu des cotisants, la structure des risques pris en charge (mesurés par les critères de l'âge, du sexe et de la morbidité) et l'efficience de la caisse. Il s'agit là d'une perte importante de compétences et de pouvoirs pour les caisses puisque le fonds de santé leur retire le droit de fixer leur taux de cotisation; les caisses perdent aussi leur rôle de gestionnaire des cotisations puisque le prélèvement est centralisé au niveau national par le Bureau fédéral de l'assurance (Bundesversicherungsamt) qui est également chargé de la péréquation entre caisses. Composé de 21 personnes parmi lesquelles trois juristes, trois économistes, un pharmacien et un médecin, ce Bureau, qui jusque-là ne comprenait que 6 salariés, a été considérablement renforcé pour faire face aux multiples enjeux de la mise en œuvre de la réforme.

Trois enjeux principaux seront développés ici. Le premier est celui du financement du système d'assurance maladie du fait non seulement de la fixation d'un montant global du budget qui lui est affecté mais aussi du changement des règles de la rémunération des médecins avec l'abandon des budgets par cabinet médical (Praxisbudget) et de la rémunération variable (en fonction du volume d'activité) qui l'accompagne, également prévus par la réforme. Le deuxième enjeu est celui de la concurrence entre caisses, dont l'intitulé de la loi annonce le renforcement, et dont les règles du jeu sont modifiées par la mise en place du Fonds et du nouveau système de péréquation à travers lequel la question de l'articulation entre concurrence et équité est également posée. Enfin, le troisième enjeu est celui de la place nouvelle occupée par l'Etat fédéral dans le système à la suite de la réforme. Si celle-ci renforce son rôle et ses compétences, l'Etat ne se substitue pas aux acteurs de l'auto-administration (Selbstverwaltung) - les caisses, les unions de médecins et les autorités en charge des hôpitaux, les Länder et les communes en particulier - mais les encadre plus étroitement en pratiquant une forme de gouvernement à distance.

\section{Encadrement et augmentation du budget de l'assurance maladie}

Avec le Fonds pour la santé (dont la mise en œuvre est prévue par la loi pour le $1^{\mathrm{er}}$ janvier 2009) est créé, de fait, un budget a priori pour l'assurance maladie dans la mesure où, d'une part, est fixé, par le gouvernement fédéral, un taux de cotisation unique pour l'année à venir ; d'autre part, le financement est complété par le budget de l'État et les cotisations des retraités et des chômeurs (dont le montant est lui aussi fixé par l'Etat). Il est également à noter qu'à partir de 2010 ce budget global sera fixé en fonction de critères de morbidité (sur la base du codage de l'activité des médecins) et non plus seulement sur des critères économiques.

Début octobre, le gouvernement, sur la base des prévisions d'un groupe d'experts (Schätzerkreis) issus du ministère de la Santé, du Bureau fédéral de l'assurance (Bundesversicherungsamt) et de la nouvelle structure fédérale des caisses légales (GKV Spitzenverband) également créée par la réforme, a fixé le montant global du taux de cotisation à 15,5\% pour 2009 (un taux de 7,3\% du salaire brut pour les salariés et les employeurs auquel s'ajoute une cotisation spéciale de 0,9\% pour les salariés). II s'agit là d'une augmentation du taux de cotisation de 0,6 point puisque, actuellement, la moyenne des taux de cotisations fixés par les différentes caisses s'établit à 14,9\%, et qui se traduit par une hausse des rentrées financières pour les caisses d'un montant de 5,8 milliards $€$. Comme le gouvernement fédéral poursuit l'objectif de réduire à terme le poids des prélèvements, elle est compensée par une diminution de 0,5 point du taux de cotisation pour l'assurance chômage et par le fait qu'à partir de 2010 les cotisations pour l'assurance maladie et l'assurance pour les soins de longue durée seront prises en compte dans le calcul de l'impôt. 
À cela s'ajoute une augmentation de la contribution financière du budget de l'Etat d'un montant d'un milliard et demi d'euros, ce qui la porte au total à 4 milliards $€$. Une augmentation annuelle de ce montant est prévue jusqu'en 2016. L'augmentation du budget global de l'assurance maladie est donc estimée à 7,3 milliards $€$. Même si ce montant pourrait diminuer du fait de la remontée du chômage due aux effets de la crise financière (et de la stagnation des salaires) il n'en reste pas moins que la mise en place du Fonds correspond à une augmentation significative du budget de l'assurance maladie, voisine de $5 \%$.

Ce choix s'explique tout d'abord par l'augmentation structurelle des dépenses de santé qui s'est traduite, pour le premier semestre 2008, par une augmentation des dépenses d'assurance maladie de $4,5 \%$, ce qui a occasionné un déficit de 944 millions $€$ pour cette période, alors que l'assurance maladie est excédentaire depuis la réforme de 2003 (Loi de modernisation : Gesundheitsmodernisierungsgesetz, GMG). Selon l'Institut de recherche pour la santé et le social IGES de Berlin (Institut für Gesundheits- und Sozialforschung), dont les prévisions annuelles servent de base aux négociations entre les caisses et les unions de médecins, une augmentation particulièrement forte est à attendre du côté des dépenses de médicaments du fait d'innovations thérapeutiques permettant d'accroître la prise en charge en ambulatoire de pathologies faisant jusqu'alors l'objet d'hospitalisations. II a prévu une augmentation de $8,1 \%$ et a préconisé la fixation d'un taux de cotisation au moins égal à 15,5\%.

La deuxième raison est que la loi de 2007 prévoit la suppression des budgets médicaux (Praxisbudgets) et le système de rémunération flottant qui l'accompagne. En 1997, chaque médecin exerçant dans le cadre de l'assurance maladie s'était vu attribuer un budget annuel tenant compte du profil moyen d'activité des médecins de sa spécialité, des caractéristiques sanitaires de sa région, de sa qualification et des spécificités de sa clientèle ; des reversements étaient prévus en cas de dépassement du budget, des versements dans le cas contraire. Selon le texte de la loi, les budgets doivent être remplacés par des volumes d'actes au montant fixé en euros (et non plus en points). La rémunération des actes médicaux ne sera donc plus fluctuante (selon le volume d'activité), mais dépendra de volumes fixés préalablement et liés au type d'activité médicale. II s'agit là d'une revendication très importante pour les médecins, qui a joué un rôle moteur dans leur mobilisation depuis l'automne 2005 (Hassenteufel, 2006).

La mise en place de ce changement de système de rémunération des médecins a été âprement négociée au cours de l'été 2008 par les représentants des caisses et des unions de médecins dans le cadre d'une Commission des rémunérations (Bewertungsausschuss). Pour trancher les différends entre les médecins et les caisses il a été fait appel, comme le prévoit la loi, à un expert 'neutre', Jürgen Wasem, pour la présider dans une composition élargie. Cette commission a tout d'abord décidé une augmentation du volume global des honoraires des médecins d'un montant de 2,7 milliards $€$, soit environ $10 \%$ de hausse (et une augmentation de $18000 €$ annuels par médecin en moyenne). Cette somme est répartie régionalement en fonction de critères de morbidité. Les augmentations les plus importantes concerneront les nouveaux Länder $(+17 \%$ en moyenne), contre $+7 \%$ en moyenne dans les autres Länder. Elle a aussi fixé le montant de la valeur unitaire du point (chaque acte médical correspond à un nombre de points selon un barème unifié) à 3,5 cents.

La mise en place de ce nouveau système ne signifie pas pour autant l'abandon de l'encadrement du volume des actes accomplis par les médecins. En effet, les budgets par cabinet médical sont remplacés par des volumes d'activés réglementaires (Regelleistungsvolumen) par médecin. La commission en a également défini les règles. Ces volumes sont calculés à partir de trois éléments : le nombre d'actes accomplis lors du trimestre précédent, l'activité moyenne des médecins de la même spécialité (lorsque l'activité est supérieure à $150 \%$ de la moyenne, la valeur du point des actes accomplis au-delà de ce niveau est
Hausse significative du budget global de l'assurance maladie...

... en prévision d'une hausse des dépenses de médicaments...

... et en réponse à l'augmentation des honoraires des médecins...

... négociée à l'été 2008

Le volume des actes reste encadré, mais selon d'autres critères 
Hausse du budget global aussi pour garantir le financement des hôpitaux

Soutien fédéral global de 3,2 milliards $€$ aux hôpitaux...

... en attendant une réforme du financement des hôpitaux baissée) et la structure par âge des patients du médecin. Enfin, il est à noter qu'un certain nombre d'actes ne sont pas inclus dans ce volume global d'honoraires (qui correspond à la fixation d'un budget a priori) déterminé par l'évolution prévue de la morbidité. II s'agit de la prévention, des prestations accomplies dans le cadre de contrats spécifiques avec les caisses (contrats prévoyant le passage par le généraliste, contrats concernant la prise en charge des affections de longue durée dans le cadre des Disease Management Programs et les contrats de santé intégrée) et des actes liés au suivi de la grossesse.

Les médecins ont donc obtenu satisfaction pour les augmentations d'honoraires (même si au départ l'Union fédérale des médecins, la Kassenärztliche Bundesvereinigung $-K B V$ - revendiquait une augmentation de 5 milliards $€$ ), l'établissement d'une valeur fixe du point et la mise hors budget d'un certain nombre d'actes, tout en acceptant la poursuite de l'encadrement du volume d'activité sous une nouvelle forme avec les volumes d'activités réglementaires.

Une troisième raison explique l'augmentation du budget global de l'assurance maladie : les difficultés financières des hôpitaux. Selon le président de la Deutsche Krankenhaus Gesellschaft -DKG (la structure qui regroupe l'ensemble des gestionnaires d'hôpitaux : Länder, communes, groupes privés, associations caritatives, et qui négocie avec les caisses), un tiers des 2100 établissements hospitaliers allemands seraient au bord de la faillite. Par ailleurs, selon le baromètre annuel publié par le Deutsches Krankenhaus Institut - la structure d'expertise, de formation et de recherche de la DKG - et établi sur la base d'un échantillon de 347 hôpitaux, un tiers d'entre eux seraient en déficit.

Cette détérioration de leur situation financière est liée, d'une part, à un encadrement budgétaire rigoureux depuis 1993 (date de la mise en œuvre de la loi de réforme structurelle de l'assurance maladie) et, d'autre part, aux importantes augmentations de salaires accordées aux médecins hospitaliers en 2006 dans le cadre de nouvelles conventions spécifiques à la suite de conflits longs et durs dans les hôpitaux gérés par les Länder et dans ceux gérés par les communes (Hassenteufel, 2006), ainsi qu'à d'autres personnels de soins. Ces augmentations ont généré des dépenses comprises entre 1,5 et 2 milliards $€$ pour 2008.

La pression sur le gouvernement fédéral et les Länder (qui gèrent les plus gros établissements, en particulier les $\mathrm{CHU}$ ) s'est accrue fin septembre avec le déroulement d'une manifestation réunissant plus de 130000 (selon la police) médecins hospitaliers, personnels de soins, étudiants en médecine et directeurs d'établissement. À la suite de cette manifestation, la ministre fédérale de la Santé, Ulla Schmidt, a notamment annoncé un soutien financier global aux hôpitaux de 3,2 milliards $€$, dont 700 millions $€$ à consacrer à l'embauche de 21000 personnels de soins supplémentaires. Le reversement de 0,5\% de leur budget sera également supprimé (ce qui correspond à une économie de 380 millions $€$ ).

Dans ce contexte, le gouvernement prépare aussi une loi réformant le financement des hôpitaux. Dans le projet de loi rendu public fin août est prévue la fin des budgets basés sur l'évolution du salaire moyen. Le financement des hôpitaux dépendra de leur activité, donc de l'évolution de la morbidité et non plus des salaires. Par ailleurs, les augmentations de salaires des médecins et des personnels de soins supérieures au salaire moyen devront être en partie financées par les caisses. Le projet prévoit également que, du fait de la généralisation de la tarification à la pathologie (Diagnosis Related Groups), en 2014 devra être mis en place un tarif fédéral unique pour chaque prestation de soins hospitaliers (avec une phase intermédiaire de passage à des tarifs régionaux). Par contre, sur la question de l'augmentation du financement des investissements dans les hôpitaux par les Länder, un accord n'a pas pu être trouvé lors de la conférence des ministres de la Santé des Länder (à laquelle participait aussi Ulla Schmidt, ministre fédérale de la Santé). 
La mise en œuvre de la réforme ne concerne pas seulement les aspects financiers, elle pose aussi la question du nouveau cadre de la concurrence entre caisses comme le souligne l'intitulé même de la loi.

\section{Quelle concurrence entre les caisses à l'avenir?}

La mise en place du Fonds pour la santé ne met pas fin à la concurrence entre les caisses au niveau des contributions financières demandées aux assurés. Malgré la mise en place d'un taux unique de cotisation, les caisses ont la possibilité de demander une prime complémentaire ("prime santé », Gesundheitsprämie) à leurs cotisants au cas où les ressources réparties par le Fonds ne suffiraient pas à couvrir leurs dépenses. Le montant de ces primes complémentaires peut aller jusqu'à $8 €$ par mois. Au-delà de ce montant s'appliquera une limite de $1 \%$ du revenu de l'assuré, avec un plafond fixé à $35 €$ par mois. À l'inverse, lorsque les caisses sont excédentaires, elles peuvent consentir des remises. Ces éléments permettent de continuer à faire jouer la concurrence entre caisses. Par ailleurs, ce nouveau modèle préserve en fin de compte le financement assurantiel tout en le centralisant et en renforçant le déséquilibre entre cotisations des assurés (du fait du forfait complémentaire que peuvent mettre en place les caisses) et des entreprises.

Par ailleurs, depuis les réformes de 2000 et 2003 , la concurrence porte aussi sur l'offre de soins du fait des possibilités données aux caisses de proposer des formes de prise en charge intégrées dans le cadre de centres de soins médicaux regroupant médecins et autres professions de santé et de verser des primes aux assurés participant à des actions de prévention et/ou à des formes de prise en charge spécifiques, en particulier le passage systématique par le généraliste (Bode, 2006). La loi de 2007 renforce les possibilités pour les caisses de contracter avec des médecins ou des groupes de médecins en dehors des accords collectifs avec les unions de médecins. Elle favorise aussi le développement d'une offre de soins ambulatoires (très) spécialisée dans les établissements hospitaliers.

Fin octobre, le Bundestag a voté la loi de développement organisationnel de l'assurance maladie légale (Gesetz zur Weiterentwicklung der Organisationsstrukturen in der GKV) afin de compléter la loi de 2007 (notamment la garantie des prestations et des honoraires en cas de faillite d'une caisse). Celle-ci prévoit la généralisation des contrats de généralistes (Hausarzt Verträge) renforçant le rôle pivot de ceux-ci et mettant en place des forfaits pour le suivi des patients (et différenciés pour les malades chroniques). Ces contrats pourront désormais être signés en dehors des unions avec un syndicat, en particulier celui des généralistes (Deutscher Hausärzte Verband), dans les Länder où le taux de syndicalisation est supérieur à $50 \%$. II s'agit là d'une impulsion nouvelle donnée à la réorganisation du système de soins allemands dans le cadre de la concurrence.

Toutefois, les caisses, qui demandaient un taux de cotisation plus élevé $(15,8 \%)$, mettent en avant la difficulté de maintenir les programmes de prise en charge spécifique de certaines maladies chroniques (des contrats pour la prise en charge d'enfants souffrant de maladies psychiques ou de malades du cancer ont été mis en cause) du fait de la mise en place du Fonds qui accroît le risque porté par les caisses et donc les incertitudes liées à sa mise en place. Par ailleurs le financement spécifique des contrats de soins intégrés, instauré par la réforme de 2003, arrive à échéance fin 2008, ce qui fait craindre la non-reconduction de la moitié d'entre eux selon le président de l'association fédérale des caisses locales (AOK). Ainsi, la mise en œuvre de la réforme pourrait entraîner une réduction de l'offre de soins.

Enfin, la réforme modifie les règles de péréquation entre les caisses, jusque-là fondées sur l'âge, le sexe et la profession des assurés, en y ajoutant la prise en
Remises ou primes supplémentaires ménagent une marge de manœuvre

Concurrence accrue sur l'offre de soins

Les généralistes peuvent dans certains cas contracter directement avec les caisses

Incertitudes sur l'avenir des contrats de soins intégrés

Un nouveau système de péréquation entre les caisses 
Fiscalisation accrue du financement de l'assurance maladie

Plus de régulation par l'Etat mais pas d'étatisation

Une responsabilité croissante confiée aux experts compte de la morbidité. Un groupe d'experts a défini 80 maladies particulièrement lourdes et à la prise en charge coûteuse qui donneront lieu à des versements complémentaires du Fonds vers les caisses afin de renforcer l'articulation entre équité et concurrence. Parmi ces pathologies, on trouve notamment le Sida, le cancer du poumon, le diabète, l'ostéoporose, l'hypertonie, l'épilepsie, la dépression... Ce nouveau système de péréquation va entraîner pour 2009 une augmentation de 2,4 milliards $€$ des reversements aux caisses locales (AOK), qui ont la structure de risque la plus défavorable, tandis que les caisses d'entreprises $(B K K)$ devront reverser 1,1 milliard € supplémentaire.

\section{Adieu à Bismarck ou gouvernement à distance ?}

La réforme de 2007 pose à nouveau la question de la remise en cause dans ses fondements mêmes du système d'assurance maladie créé dans les années 1880. En effet, plusieurs évolutions, renforcées par la dernière réforme, tendent à rapprocher le système d'assurance maladie allemand d'un système national de santé géré directement par l'Etat (Hassenteufel, Palier, 2007).

II s'agit tout d'abord de la fiscalisation accrue du financement, qui cependant ne s'élève aujourd'hui qu'à environ $10 \%$ du financement de l'assurance maladie (contre plus de $45 \%$ en France du fait de la CSG). On peut ensuite mettre en avant le principe de l'assurance universelle posé par la loi puisque tout assuré sorti du système légal d'assurance maladie a désormais le droit d'y retourner. Environ la moitié des personnes sans couverture maladie (soit quelque 100000 personnes) a ainsi été réintégrée depuis l'adoption de la loi de 2007. Cependant, la réforme n'a pas remis en cause la séparation entre assurance maladie légale et assurance privée puisque l'obligation de cotiser pour le système légal (public) ne concerne que les personnes dont le revenu mensuel brut est inférieur à $4000 €$ (celles dont le revenu est supérieur à ce niveau peuvent s'assurer dans le privé, ce qui est le cas d'environ $7 \%$ des assurés). La concurrence entre caisses privées et caisses légales ne concerne donc que ces assurés ; pour les autres (l'essentiel de la population) le libre choix de la caisse se limite au système public.

La réforme correspond surtout à un encadrement budgétaire accru du système maladie par l'Etat. Cette logique avait été introduite par la loi de 1992 mais, avec le Fonds, se met en place une fixation a priori du budget de l'ensemble de l'assurance maladie (Paquet, 2007). Ainsi se trouve renforcé le rôle de l'Etat fédéral dans un système traditionnellement auto-administré par la négociation collective entre les caisses, les médecins et les gestionnaires d'hôpitaux. Toutefois, il s'agit là plus de l'avènement d'un Etat régulateur, comme dans d'autres systèmes de protection maladie européens (Hassenteufel, Smyrl, 2008), que d'une étatisation de l'assurance maladie. En effet, même si non seulement le ministère fédéral de la Santé (et plus généralement le gouvernement fédéral qui établit désormais le niveau des cotisations) et d'autres structures étatiques fédérales (en particulier le Bureau fédéral de l'assurance) jouent un rôle accru, ils ne se substituent pas complètement aux caisses, aux médecins et aux Länder qui continuent à négocier, notamment sur le montant des honoraires et leur répartition.

Cette négociation est cependant plus fortement encadrée et arbitrée par des experts nommés par le gouvernement fédéral. C'est ce que montre la mise en place du comité d'experts qui a proposé le niveau du taux de cotisation décidé par le gouvernement fédéral ou l'élargissement de la commission des rémunérations à un expert pour trancher les différends (ce qui a notamment permis l'adoption de la valeur du point pour la rémunération des actes médicaux).

On peut également souligner le rôle croissant joué par l'Institut für Qualität und Wirtschaftlichkeit im Gesundheitswesen (IQWIG) qui définit les normes thérapeutiques et les outils d'évaluation de l'efficacité et de la qualité des soins et 
des médicaments. Cette agence d'expertise est financée par l'assurance maladie, mais le ministère fédéral de la Santé est fortement représenté au sein de son comité directeur (aux côtés des acteurs de l'auto-administration).

Remarquons, enfin, que la Commission fédérale commune (Gemeinsamer Bundesausschuss), réunissant les représentants des différents acteurs de l'assurance maladie (caisses, médecins, hôpitaux), est désormais présidée par une personnalité 'neutre' et que ses membres le sont maintenant à plein temps. Elle définit les prestations prises en charge par l'assurance maladie et travaille de ce fait en lien étroit avec l'IQWIG.

Par ces différentes évolutions institutionnelles, les acteurs de l'auto-administration sont plus fortement intégrés à l'État et agissent de façon croissante par délégation pour lui et en fonction des objectifs qu'il leur fixe. Parmi ces acteurs délégués du gouvernement à distance de l'assurance maladie, on peut ajouter la nouvelle structure fédérale de l'ensemble des caisses (le GKV Spitzenverband qui a une centaine de salariés et qui est de droit public), dont la création par la loi de 2007 réduit considérablement le rôle des structures fédérales de chaque caisse qui ne seront plus des organisations de droit public à partir du $1^{\text {er }}$ janvier 2009. On assiste donc plutôt à un encadrement croissant de l'autoadministration correspondant à un gouvernement à distance par l'Etat fédéral, qui fixe des règles du jeu de plus en plus contraignantes et qui s'est doté de moyens de surveillance, de contrôle, d'évaluation et de sanction accrus sur l'ensemble des acteurs de l'assurance maladie.

ON COMPREND DONC QUE LES CAISSES RESTENT TRES CRITIQUES vis-à-vis de cette réforme à laquelle elles avaient essayé de s'opposer, et ceci d'autant plus que les médecins ont été plutôt les gagnants des négociations en obtenant des augmentations d'honoraires importantes. Ceux-ci continuent toutefois à également dénoncer la "bureaucratisation » et la centralisation du système d'assurance maladie du fait de la mise en place du Fonds. Ces nombreuses critiques de la loi de 2007 ont un certain impact dans l'opinion publique, comme en témoigne un sondage réalisé en mars 2008 auprès de 2000 personnes par l'institut Forsa pour la caisse des techniciens : $66 \%$ des personnes interrogées pensent que la réforme n'apporte pas d'amélioration au système de santé ; $74 \%$ pensent que le Fonds augmente la bureaucratie ; $72 \%$ estiment qu'il y aura de ce fait moins de concurrence entre les caisses et $80 \%$ pensent que l'Etat réduira sa contribution au détriment de la prise en charge des soins. Le contexte économique fortement dégradé par la crise financière risque d'attiser les craintes et le scepticisme sur les effets de la réforme de l'assurance maladie de 2007 qui sera très probablement... réformée après les élections de 2009 !

\section{Indications bibliographiques}

BODE I., «Financement solidaire et gouvernance concurrentielle. Le modèle allemand d'organisation de la santé en débat », Revue française des Affaires sociales, vol.60 n2-3 (2006)

BouRgeols I., “Assurance maladie : bientôt un 'Fonds santé' ? », Regards sur l'économie allemande, $n^{\circ} 77$, juillet 2006

«Gesundheitspolitik : Fehler beseitigen - weitere Schritte unerlässlich », SACHVERSTÄNDIGENRAT ZUR BEGUTACHTUNG DER GESAMTWIRTSCHAFTLICHEN ENTWICKLUNG, Jahresgutachten 2008/09, pp. 386-406

HAssenteufel P., « Libéralisation ou étatisation de l'assurance maladie ? », Regards sur l'économie allemande, $\mathrm{n}^{\circ} 79$, décembre 2006

Hassenteufel P., Palier B., « Towards Neo-Bismarckian Health Care States ? Comparing Health Insurance Reforms in Bismarckian Welfare Systems », Social Policy and Administration, 16 (4), 2007

HASSENTEUfEl P., SmyRl M., « Explaining Governance Changes in Health Care SystemsThe role of programmatic actors », texte présenté au workshop « Explaining Health Care System Change ", Université de Brême, décembre 2008

PAQUET R., “ Der Abschied von der Sozialversicherung beginnt », Gesundheitspolitischer Informationsdienst, 3 (1), mai 2007
Un gouvernement à distance via des acteurs délégués 